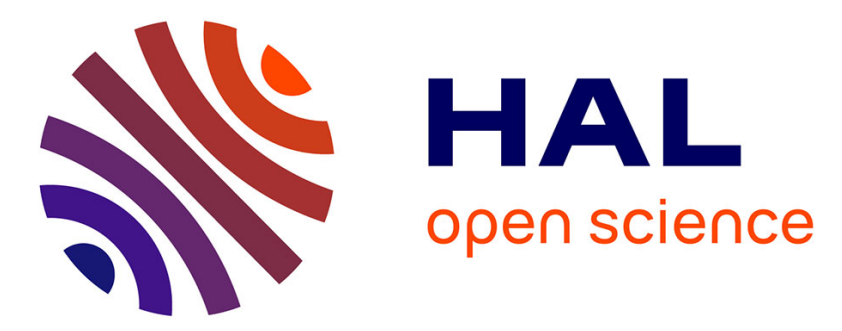

\title{
Uniaxial Negative Thermal Expansion in Polymorphic 2-Bromobenzophenone, Due to Aromatic Interactions?
}

Michela Romanini, Ivo Rietveld, Maria Barrio, Philippe Négrier, Denise Mondieig, Roberto Macovez, René Céolin, Josep-Lluís Tamarit

\section{To cite this version:}

Michela Romanini, Ivo Rietveld, Maria Barrio, Philippe Négrier, Denise Mondieig, et al.. Uniaxial Negative Thermal Expansion in Polymorphic 2-Bromobenzophenone, Due to Aromatic Interactions?. Crystal Growth \& Design, 2021, 21 (4), pp.2167-2175. 10.1021/acs.cgd.0c01603 . hal-03258007

\section{HAL Id: hal-03258007 https://hal.science/hal-03258007}

Submitted on 11 Jun 2021

HAL is a multi-disciplinary open access archive for the deposit and dissemination of scientific research documents, whether they are published or not. The documents may come from teaching and research institutions in France or abroad, or from public or private research centers.
L'archive ouverte pluridisciplinaire $\mathbf{H A L}$, est destinée au dépôt et à la diffusion de documents scientifiques de niveau recherche, publiés ou non, émanant des établissements d'enseignement et de recherche français ou étrangers, des laboratoires publics ou privés. 


\section{Uniaxial negative thermal expansion in polymorphic 2-bromobenzophenone, due to aromatic interactions?}

Michela Romanini ${ }^{1}$, Ivo. B. Rietveld ${ }^{2,3,}{ }^{*}$, Maria Barrio $^{4}$, Philippe Negrier ${ }^{5}$, Denise Mondieig ${ }^{5}$, Roberto Macovez ${ }^{4}$, René Céolin ${ }^{4}$, Josep-Lluís Tamarit ${ }^{4, * *}$

${ }^{1}$ Departament de Física de la Matèria Condensada, Facultat de Física, Universitat de Barcelona, Diagonal 647, E-08028 Barcelona, Catalonia, Spain ${ }^{2}$ SMS Laboratory (EA 3233), Université de Rouen-Normandie, Place Émile Blondel, Mont Saint Aignan 76821, France

${ }^{3}$ Faculté de Pharmacie, Université de Paris, 4 avenue de l'observatoire, 75006, Paris, France

${ }^{4}$ Grup de Caracterizació de Materials, Departament de Fisica, EEBE and Barcelona Research Center in Multiscale Science and Engineering, Universitat Politècnica de Catalunya, Eduard Maristany, 10-14, 08019 Barcelona, Catalonia, Spain

${ }^{5}$ Université de Bordeaux, LOMA, UMR 5798, CNRS, F-33400 Talence, France

*ivo.rietveld@univ-rouen.fr, current address: Neutron Science Laboratory, Institute for Solid State Physics, University of Tokyo, 5-1-5 Kashiwanoha, Kashiwa, Chiba 277-8581, Japan

** josep.lluis.tamarit@upc.edu 


\section{Abstract}

The structure of the metastable form II of 2-bromobenzophenone, obtained by crystallization from the melt, has been determined by powder X-ray diffraction. Form II has been solved in the centrosymmetric monoclinic space group $P 21 / c$ with $a=8.4896(19) \AA, b=6.5438(8) \AA, c=$ $20.253(1) \AA ; \beta=104.452(6)^{\circ}$ and $Z=4\left(Z^{\prime}=1\right)$ at $200 \mathrm{~K}$. Both form I and form II contain a multitude of aromatic interactions and the strength and direction of these interactions could only be interpreted with the support of the thermal expansion tensors. Both forms exhibit, unexpectedly, uniaxial negative thermal expansion, while hydrogen bonding does not play a significant role in either of these two structures. It appears to be the first time in the literature that uniaxial negative thermal expansion may be caused by aromatic interactions. Thermodynamic properties at normal and high pressure have been determined for the stable and metastable phases and a pressure-temperature phase diagram has been constructed. While the metastable form behaves monotropically with respect to the stable form under ambient conditions, the phase relationship becomes enantiotropic at high pressure, providing a clear example of phase behavior in which the densest form is not the most stable form under ambient conditions. 


\section{INTRODUCTION}

Benzophenone (chemical formula $\mathrm{C}_{13} \mathrm{H}_{10} \mathrm{O}$, Figure 1) and its derivatives exhibit great diversity in biochemical and photophysical properties, which are exploited in a huge number of applications. They are inhibitors of enzymes and possess antimicrobial, anticancer, and antiviral properties. Moreover, they are good absorbers of ultraviolet light and are used to prevent damage in the skin in sunscreen lotions and to avoid photo-degradation of polymers for packaging. Benzophenone derivatives are also used as crosslinking units between polymers, and as photoinitiators among others. ${ }^{1-6}$ In pharmaceutical chemistry, the benzophenone scaffold is a basic synthetic building block. ${ }^{7}$ Finally, these molecules are used in light-emitting diodes and in other optoelectronic applications due to their linear and nonlinear optical properties. ${ }^{8,9}$

Benzophenone (BPh) was observed to exhibit polymorphism in the latter part of the $19^{\text {th }}$ century ${ }^{10}$ and a short historical review has been given in a paper by Kutzke et al. ${ }^{11}$ The polymorphism is strongly related to the chiral character of the benzophenone molecule. The chirality is associated with the dihedral angle between the two planes defined by the phenyl rings, which can also be described in terms of the angles of either phenyl group with the central ketone plane, $>\mathrm{C}-(\mathrm{C}=\mathrm{O})-\mathrm{C}<$, through the two torsion angles $\mathrm{O}=\mathrm{C}-\mathrm{C}-\mathrm{C}$, in which the last carbon atom is part of either one of the phenyl rings. However, the enantiomers (i.e. stereoisomers that cannot be superposed on their mirror image) only materialize in the solid state as both in the liquid and gas phase, the aromatic rings of BPh can turn more or less freely resulting in a dynamically averaged single conformation.

Whereas the crystal structure of the stable polymorph of BPh (orthorhombic, space group $P 2{ }_{1} 2_{1} 2_{1}$ ) contains only one enantiomer, ${ }^{12}$ the metastable polymorph (monoclinic, space group $C 2 / c)$, obtained by recrystallization from the liquid, which is by its nature racemic, is a racemate. ${ }^{11}$ In addition, the two polymorphs differ in their molecular conformation characterized by the dihedral angle between the planes of the two phenyl rings, while all other bond lengths and angles remain similar. In the stable phase of BPh, this dihedral angle is $54.4^{\circ}$, whereas in the metastable phase it is $64.5^{\circ} .{ }^{11}$ Both dihedral angles are close to the theoretical optimum angle of $52.5^{\circ}$ calculated for the isolated molecule using DFT. ${ }^{13}$

Because the metastable form of BPh is racemic, a transition on heating from this phase to the enantiomer-pure, stable form would imply a chiral inversion of one of the two enantiomers. This process has not been reported for BPh as it was for 4-methylbenzophenone (Figure 1), ${ }^{14}$ which may imply that the activation energy for the conversion is too high. Ma and Coppens studied ten supramolecular crystals in which BPh is encapsulated as a guest and determined the distortion energy of the different conformations of BPh as a function of the dihedral angle between the phenyl planes ranging between 42 and $68^{\circ}{ }^{13}$ They obtained energy differences of up to 25 $\mathrm{kJ} \cdot \mathrm{mol}^{-1}$, which is significant compared to the lattice energy of the stable, $-85.9 \mathrm{~kJ} \cdot \mathrm{mol}^{-1}$, and metastable, $-83.0 \mathrm{~kJ} \cdot \mathrm{mol}^{-1}$, polymorphs. ${ }^{11}$ Quantum chemical calculations for BPh on the rotational potential energy surface showed that the dihedral angle between the phenyl rings remains near $60^{\circ}$ along most of the minimum rotational energy path, thus, close to the values found in both stable and metastable structures. This result suggests that steric interactions are more important than conjugation and crystal-packing forces when it comes to the molecular conformation. ${ }^{15}$ 
The interplay of chirality and polymorphism in the BPh derivatives is diverse. In this work, a study on 2-bromobenzophenone (2-BrBPh, Figure 1 ) is presented. Introducing a bromine atom at the 2-position causes stronger steric hindrance between the aryl groups in comparison to unsubstituted BPh. The structure of the stable phase (form I) of 2-BrBPh was determined by Baumer et al. in 2005 through single crystal X-ray diffraction and was found to be a racemate (monoclinic, space group P2 $1 / a$ ). ${ }^{16}$ A new metastable phase (form II), obtained by recrystallization from the supercooled liquid, was recently discovered by Baran et al., but its structure remains to be determined. ${ }^{17}$ Baumer et al., through DFT calculations and quantum chemical modelling on the isolated molecule, demonstrated the existence of two low-energy conformers with different torsion angles (both existing as a pair of enantiomers), while the conformation with the lowest energy was very close to the observed torsion angle in phase I. Because the energy barrier between the two conformations was found to be small, the gas phase at RT should contain about $34 \%$ of the other conformation. Thus, they concluded that the crystal packing has a "substantial influence on the conformational parameters" (i.e. the torsion angle) of the 2-BrBPh molecule in the crystalline state. ${ }^{16}$ However, this must be within the boundaries created by the steric hindrance between the two rings and the bromine atom.

It is interesting to point out that the equilibrium conformation of unsubstituted benzophenone is a "helicoidal" conformer with both phenyl rings tilted with respect to the plane of the carbonyl group. ${ }^{18}$ Such a geometry favours cross-conjugation between both equivalent phenyl moieties by sacrificing the optimum coplanar geometry which would maximize electronic conjugation of a single phenyl ring with the central carbonyl group. In contrast, in the brominated 2-BrBPh derivative the phenyl ring is coplanar with the carbonyl group. This entails that the large electronegativity of the $\mathrm{Br}$ substituent leads to a strong molecular asymmetry which suppresses the energy gain of cross-conjugation between the two aryl moieties, leading to a coplanar geometry that maximizes electron delocalization between the carbonyl group and the unsubstituted ring.

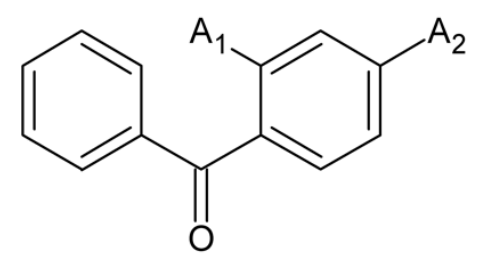

Figure 1. Molecular structure of benzophenone $\left(A_{1}=A_{2}=\right.$ ' $H$ ' $)$, 4-methylbenzophenone $\left(A_{1}=\right.$ ' $H$ ', $\left.A_{2}={ }^{\prime} \mathrm{CH}_{3}{ }^{\prime}\right)$, 2-bromobenzophenone $\left(A_{1}={ }^{\prime} \mathrm{Br}^{\prime}, \mathrm{A}_{2}={ }^{\prime} \mathrm{H}{ }^{\prime}\right)$, 2-chlorobenzophenone $\left(\mathrm{A}_{1}={ }^{\prime} \mathrm{Cl}, \mathrm{A}_{2}=\right.$ ' $H$ '), 4-bromobenzophenone $\left(A_{1}={ }^{\prime} H\right.$ ', $\left.A_{2}={ }^{\prime} B r^{\prime}\right)$

A survey of other mono-substituted analogues (Figure 1), such as 2-chlorobenzophenone (2$\mathrm{ClBPh}),{ }^{19} 4$-bromobenzophenone $(4-\mathrm{BrBPh}),{ }^{20}$ or 4 -methylbenzophenone $(4-\mathrm{methylBPh})^{14}$ for which crystal structures of the stable and metastable phases have been described, shows that the interplay between chirality, the structures, and their stability is highly unpredictable. Whereas stable phases of 4-methylBPh $\left(P 2_{1} / C\right), 2$-ClBPh $\left(P 2_{1} / C\right)$ and 4-BrBPh $\left(P 2_{1} / C\right)$ are centrosymmetric (racemic) crystal structures, metastable phases (when they have been discovered) were found either to contain both enantiomers, i.e. to be racemic crystals, in the case of 4-BrBPh with a $P-1$ space group, or to contain only one enantiomer, i.e. to be enantiomorph crystals, in the case of 4-methylBPh with a $P 3_{1}$ space group. Interestingly, for the 
unsubstituted $\mathrm{BPh}$, the stable form contains only one enantiomer in the $P 2{ }_{1} 2_{1} 2_{1}$ space group, while the metastable form is the racemic crystal with the space group $C 2 / c$. Thus, Wallach's rule $^{21}$ claiming that racemic crystals have in general a higher density and stability does not seem to be universally true, in particular in the case of BPh.

Intermolecular interactions caused by aromatic systems have received considerable attention. ${ }^{22,23}$ Often, these interactions are called $\pi-\pi$ stacking and three main configurations between two aromatic rings are identified: ${ }^{23}$ i) face-to-face, ii) parallel-displaced, and iii) Tshaped edge-to-face (also designated as $\mathrm{C}-\mathrm{H} \cdots \pi$ ). Nonetheless, the interpretation of the strengths of these quite different interaction geometries is still not fully understood, while the use of the term $\pi-\pi$ stacking to describe aromatic interactions is being advised against. ${ }^{24,25}$ In particular for small, simple molecules such as benzene direct face-to-face stacking does not increase the interaction between two aromatic rings. ${ }^{25}$ However, larger sized aromatic molecules, electron withdrawing substituents on aromatic rings, parallel-displaced geometries and the T-shaped geometries all lead to more favorable interactions for which the label 'aromatic interaction' is probably too general. ${ }^{23-25}$

In addition to the problem of the identity of 'the' aromatic interaction, the directionality of the aromatic interactions is not as straightforward as for example for hydrogen bonds. Thermal expansion studies may allow to obtain information about the directionality; however, such studies related to molecules that are mainly aromatic in nature are fairly rare. The aromatic molecule 1-hydroxypyrene was found to exhibit positive thermal expansion in all directions. ${ }^{26}$ Nonetheless, Saraswatula et al. demonstrate that increasing the strength of the aromatic interactions can decrease thermal expansion in a series of similar aromatic molecules. ${ }^{27}$ Glipizide is probably one of the earlier examples demonstrating uniaxial negative thermal expansion (NTE), although steric hindrance appears to be the cause of the uniaxial contraction on heating. ${ }^{28}$ NTE has been observed for co-crystals with 4-phenylazopyridine, which was ascribed to a rotational movement of the molecules and to the hydrogen bonds present in the co-crystals. ${ }^{29}$ Furthermore, NTE has been shown to be generated by oppositely shifting molecular layers, although hydrogen bonding was again involved in this case..$^{30,31}$ It is therefore, up to now, not clear whether aromatic interactions are able to directly cause (uniaxial) NTE as hydrogen bonds are known to be capable of.

In the following, the crystal structures of 2-bromobenzophenone are analyzed. It is demonstrated that, despite the existence of two possible conformers for each enantiomer of $2 \mathrm{BrBPh}$, both stable and metastable crystalline phases belong to a centrosymmetric space group and thus, both enantiomers are present. These enantiomers only favor one conformation. Moreover, temperature-resolved X-ray diffraction demonstrates the presence of uniaxial NTE in the presence of mainly aromatic interactions. It is shown that the density of the metastable phase is higher than that of the stable phase and its consequences on the stability relationship between phases I and II as a function of pressure will be analyzed.

\section{EXPERIMENTAL SECTION}

Material. 2-bromobenzophenone ((2-bromophenyl)phenylmethanone) was purchased from Aldrich with a purity of $98 \%$. Most of the experiments were performed after careful purification 
of the sample through recrystallization in ethanol by the group of Professor A. Jezowski at the Institute of Low Temperature and Structure Research, Wroclaw (Poland).

Differential Scanning Calorimetry. Differential scanning calorimetry (DSC) experiments were carried out with a conventional Q100 thermal analyzer from TA Instruments. It was calibrated using the melting point of indium ( $\left(T_{\text {fus }}=429.75 \mathrm{~K}\right.$ and $\Delta_{\text {fus }} \mathrm{H}=3.267 \mathrm{~kJ} \mathrm{~mol}^{-1}$ ). The specimens with sample masses around $10 \mathrm{mg}$ were weighed with a microbalance sensitive to $0.01 \mathrm{mg}$ and sealed in aluminum pans.

High-pressure differential thermal analysis. High-pressure differential thermal analysis (HP-DTA) measurements were carried out with an in-house-constructed apparatus similar to the equipment built by Würflinger ${ }^{32}$ operating between 0-300 MPa. Melting temperatures as a function of pressure were determined of specimens mixed with an inert Perfluorinated liquid (Galden; Bioblock Scientifics, Illkirch, France) to eliminate air while enclosed in tin capsules. The onset of the calorimetric peaks was taken as the melting temperature.

High resolution X-ray diffraction as a function of temperature. High-resolution Xray diffraction as a function of temperature was performed at normal pressure using DebyeScherrer geometry and transmission mode. $C u K \alpha_{1}(\lambda=1.54056 \AA)$ radiation was used in a horizontally mounted INEL diffractometer with a quartz monochromator and a cylindrical position-sensitive detector (CPS-120) with 4096 channels ( $0.029^{\circ} 2 \theta$-angular step). Powder samples were introduced into a $0.5-\mathrm{mm}$-diameter Lindemann capillary. The temperature was controlled with a 700 series Oxford Cryostream Cooler from Oxford UK Cryosystems.

Lattice parameters as a function of the temperature were determined through isothermal acquisition of $\mathrm{X}$-ray patterns between $100 \mathrm{~K}$ and the respective melting points of both forms. External calibration by means of the cubic phase $\mathrm{Na}_{2} \mathrm{Ca}_{3} \mathrm{Al}_{2} \mathrm{~F}_{4}$ and cubic spline fitting was used to convert the measurement channels into $2 \theta$. Peak positions were determined using pseudo-Voigt fitting.

Liquid density measurements. The density of the (supercooled) liquid was determined as a function of the temperature with a DMA-5000 Density Meter from Anton Paar. Data were obtained at isothermal steps on cooling from $363.2 \mathrm{~K}$ down to $298.2 \mathrm{~K}$ with a temperature control of $\pm 1 \mathrm{mK}$.

\section{RESULTS AND DISCUSSION}

\section{Characterization of the polymorphic behavior}

Thermodynamic study. A DSC curve of a 2-BrBPh sample in a closed pan is presented in Figure 2. Heating from room temperature, the sample (initially purified form I) exhibited a single endothermic effect ascribed to a melting transition with a mean onset at $312.2 \pm 0.5 \mathrm{~K}$ and a mean value of the melting enthalpy of $18.7 \pm 0.4 \mathrm{~kJ} \cdot \mathrm{mol}^{-1}$ (see Table 1 ). On reheating after quick or slow cooling of the melt to $193 \mathrm{~K}$, a glass transition was observed at $224.8 \pm 0.5 \mathrm{~K}$ (midpoint), followed by an exothermic peak at around $265 \mathrm{~K}$ corresponding to the recrystallization of the 
supercooled liquid into the metastable form II, which melts at $302.2 \pm 0.5 \mathrm{~K}$, with a lower melting enthalpy, $16.3 \pm 0.7 \mathrm{~kJ} \cdot \mathrm{mol}^{-1}$, than form I.

Table 1. Enthalpy $(\Delta H)$, entropy $(\Delta S)$, and volume $(\Delta v)$ changes at the observed phase transitions together with the experimental $\left((\mathrm{d} T / \mathrm{d} P)^{\exp }\right)$ and calculated $\left((\mathrm{d} T / \mathrm{d} P)^{\text {calc }}\right)$ slopes of the melting temperatures with respect to the pressure

\begin{tabular}{lccc}
\hline $\begin{array}{l}\text { Property / Phase } \\
\text { transition }\end{array}$ & $\mathrm{I} \rightarrow \mathrm{L}$ & $\mathrm{I} \rightarrow \mathrm{L}$ & $\mathrm{I} \rightarrow \mathrm{I}^{*}$ \\
\hline$T / \mathrm{K}$ & $312.2 \pm 0.5$ & $302.2 \pm 0.5$ & \\
\hline$\Delta H / \mathrm{J} \cdot \mathrm{mol}^{-1}$ & $18.7 \pm 0.4$ & $16.3 \pm 0.7$ & $2.4 \pm 0.9$ \\
\hline$\Delta S / \mathrm{J} \cdot \mathrm{K}^{-1} \cdot \mathrm{mol}^{-1}$ & $59.8 \pm 1.3$ & $53.8 \pm 2.3$ & $6 \pm 3$ \\
\hline$\Delta v / \mathrm{cm}^{3} \cdot \mathrm{mol}^{-1}$ & $17.69 \pm 0.13$ & $17.45 \pm 0.16$ & -0.0019 \\
& & & \pm 0.0025 \\
\hline$(\mathrm{d} T / \mathrm{d} P)^{\mathrm{calc}} /{\mathrm{K} \cdot \mathrm{MPa}^{-1}}$ & $0.296 \pm 0.009$ & $0.324 \pm 0.017$ & -0.0003 \\
& & & \pm 0.0005 \\
\hline$(\mathrm{d} T / \mathrm{d} P)^{\exp } / \mathrm{K} \cdot \mathrm{MPa}^{-1}$ & $0.290 \pm 0.012$ & $0.322 \pm 0.012$ & - \\
\hline
\end{tabular}

* Values for the I to II transition have been calculated; the enthalpy and entropy differences are in principle valid at $307.2 \mathrm{~K}$ (the mean of the two melting temperatures), $\Delta v$ has been evaluated at $307.2 \mathrm{~K}$ leading to a dT/dP slope evaluated at the same temperature. Although the errors are in certain cases larger than the values themselves, the volume difference and the slope must have negative values (see eqs. 1 and text on relative stability below).

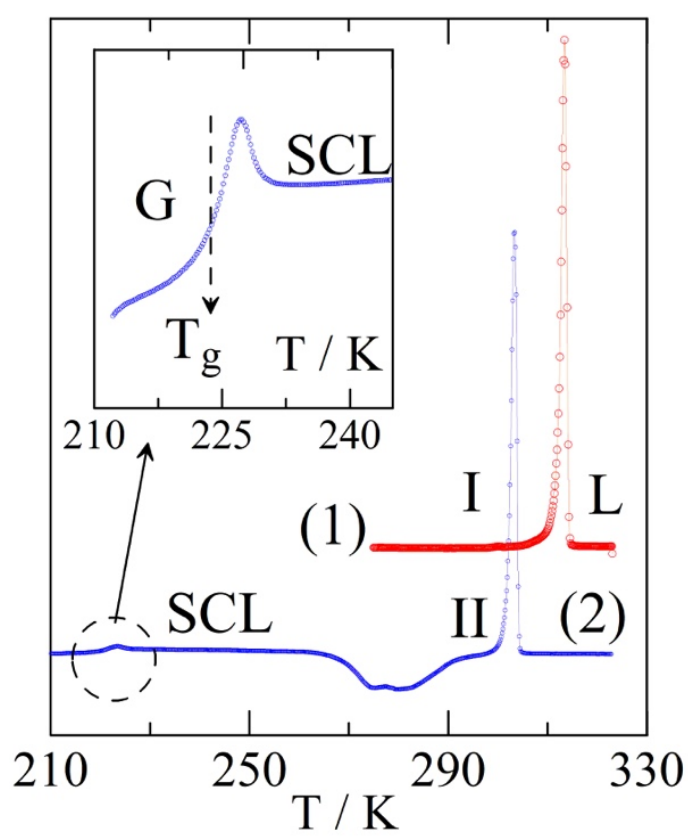

Figure 2. Differential scanning calorimetry curves of 2-bromobenzophenone recorded at a rate of $10 \mathrm{~K} \mathrm{~min}^{-1}$. (1) First heating of the purified form I. (2) Second heating after cooling the melt to $193 \mathrm{~K}$ in which a glass transition at $T_{\mathrm{g}}$ (from the glass state, $\mathrm{G}$, to the supercooled liquid, $\mathrm{SCL}$ ) can be observed (see inset). 
Crystallographic study. Powder X-ray diffraction (PXRD) patterns of form I as a function of the temperature from $100 \mathrm{~K}$ to its melting point have been fitted to the published structure ${ }^{16}$ and virtually the same results were obtained. An example of the PXRD at room temperature together with the Rietveld refinement is provided in Figure S1. The structure of form I, which contains $Z=4$ molecules in the unit cell, with only one molecule in the asymmetric unit $\left(Z^{\prime}=1\right)$, was reported in the space group $P 2_{1} / a .{ }^{16}$ For the sake of comparison with the structure of form $\mathrm{II}$, the structure can be transformed from the space group $P 2_{1} / a(a, b, c, \beta)$ to the space group $P 2_{1} / c\left(a^{\prime}, b, c^{\prime}, \beta\right)\left(a^{\prime}=c\right.$ and $\left.c^{\prime}=a\right)$.

Although form II was recently discovered by Baran et al., ${ }^{17}$ the structure and its relative stability with respect to form I have not been resolved. In order to solve the structure, a crystalline powder of form I was introduced into a Lindemann capillary and subjected to the described thermal treatment (see Figure 2). Growth of form II occurred at $280 \mathrm{~K}$. Long acquisition-time high-resolution PXRD patterns were acquired at $200 \mathrm{~K}$ as well as at room temperature (293 K). The lattice was indexed using the X-cell software ${ }^{33}$ followed by a Pawley profile-fit minimizing the $R$-factor, $R_{\text {wp }},{ }^{34}$ to confirm the space group $\left(P 2_{1} / C\right)$. This procedure enables to refine the lattice parameters, zero-shift, background, and peak profile parameters. To fully determine the structure of form II, rigid body Rietveld refinement was used based on the molecule in form I, thus all bond lengths, including those with hydrogen, are based on the existing molecular geometry of form I. In the Rietveld refinement therefore, only the angles between the cycles and the carbonyl group were adjusted. The fitted diffraction pattern is presented in Figure 3. Results of the Rietveld refinement of form II have been compiled in Table $\mathbf{2}$ together with the data of form I. A list of refinement parameters for form II can be found in Table S1 in the Supporting Information.

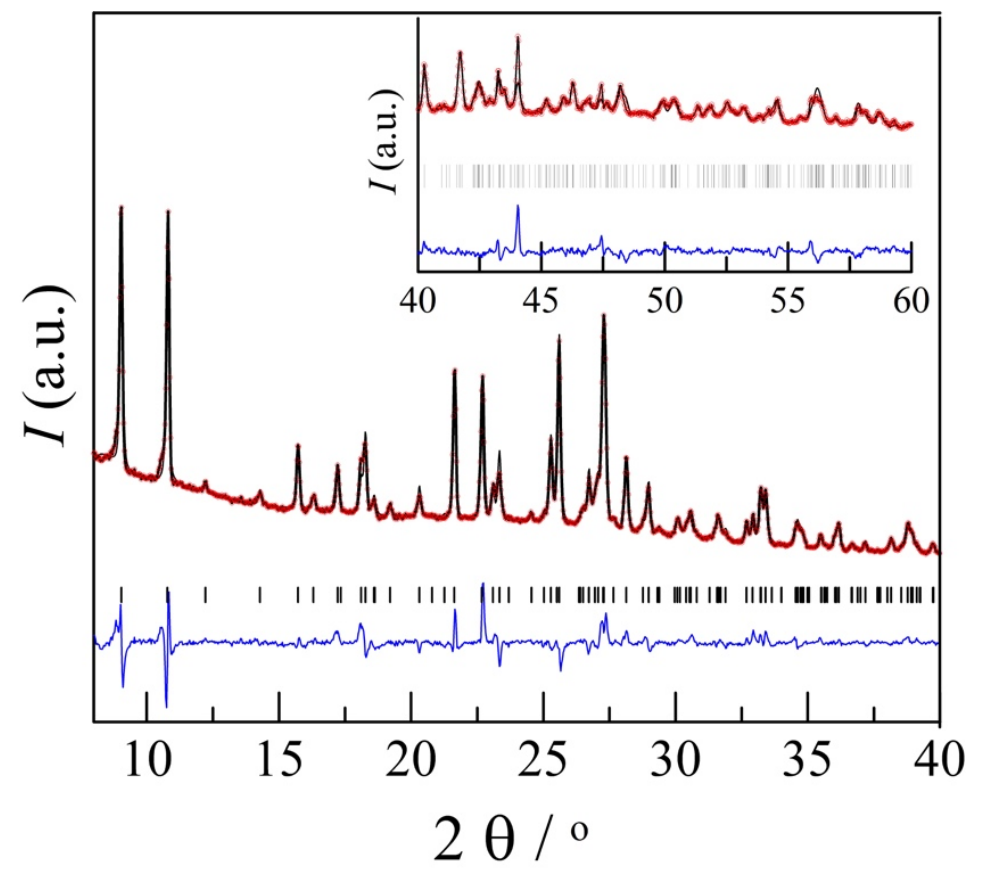

Figure 3. Experimental (red circles) and Rietveld refined (black line) diffraction patterns along with the difference profile (blue line) and Bragg reflections (vertical bars) of the monoclinic $P 2_{1} / \mathrm{C}$ space group metastable phase II of 2-Br-benzophenone at $200 \mathrm{~K}$. The inset corresponds to a $4.5-$ times-magnified intensity scale in the $40-60^{\circ} 2 \theta$ range for clarity. 
Table 2. Results of the Rietveld refinements of 2-Br-BPh form I and form II

\begin{tabular}{lcc}
\hline Phase & form I & form II \\
\hline Formula & $\mathrm{C}_{13} \mathrm{H}_{9} \mathrm{BrO}$ & $\mathrm{C}_{13} \mathrm{H}_{9} \mathrm{BrO}$ \\
\hline $\mathrm{FW}\left(\mathrm{g} \cdot \mathrm{mol}^{-1}\right)$ & 261.118 & 261.118 \\
\hline Crystal system & Monoclinic & Monoclinic \\
\hline Space group & $P 2_{1} / a$ & $P 2_{1} / \mathrm{c}$ \\
\hline$T(\mathrm{~K})$ & $200(1)$ & $200(1)$ \\
\hline Wavelength $(\AA)$ & 1.54056 & 1.54056 \\
\hline & $a=7.8160(3) \AA$ & $a=8.4896(19) \AA$ \\
\cline { 2 - 3 } Unit cell & $b=16.7374(7) \AA$ & $b=6.5438(8) \AA$ \\
dimensions & $c=8.4654(4) \AA$ & $c=20.253(1) \AA$ \\
\cline { 2 - 3 } & $\alpha=90^{\circ}$ & $\alpha=90^{\circ}$ \\
\hline$Z\left(Z^{\prime}\right)$ & $\beta=97.610(4)^{\circ}$ & $\beta=104.452(6)^{\circ}$ \\
\hline$V\left(\AA^{3}\right)$ & $\gamma=90^{\circ}$ & $\gamma=90^{\circ}$ \\
\hline$D_{\mathrm{x}}\left(\mathrm{g} \cdot \mathrm{cm}^{-3}\right)$ & $4(1)$ & $4(1)$ \\
\hline$R_{\mathrm{wp}}(\%)$ & $1097.68(8)$ & $1089.5(4)$ \\
\hline$R_{\mathrm{p}}(\%)$ & $1.5801(1)$ & $1.5918(6)$ \\
\hline
\end{tabular}

${ }^{\text {a }}$ Form $\mathrm{I}$ is refined following Baumer et al. ${ }^{16}$ using data obtained on the same equipment as the data for form II.

For both polymorphs I and II, the space group is centrosymmetric with a single molecule in the asymmetric unit $\left(Z^{\prime}=1\right)$. Both phases have therefore an inversion center, which allows the crystallographic transformation within the structure from one enantiomer to the other.

The Cambridge Crystallographic Data Centre deposit (CCDC 2023202) contains the supplementary crystallographic data for this paper. It can be obtained free of charge from the CCDC via www.ccdc.cam.ac.uk/data_request/cif.

The molecular conformations within both phases are quite similar. The subtle differences are related to the relative position of the planes of the phenyl groups towards each other through the torsion angles containing the carbonyl group (see Table 3). For 01-C1-C1A-C6A, the bromine substituted phenyl group, the angles are $68.3(5)^{\circ}$ and $66.8(10)^{\circ}$ and for 01-C1-C1B-C6B, the unsubstituted phenyl group, the angles are $17.6(6)^{\circ}$ and $28.8(9)^{\circ}$ for the stable form I and the metastable form II, respectively (see the inset of Figure $\mathbf{5}$ for the atom labels). As indicated by Baumer et al., these angles demonstrate the highly asymmetric character of 2-BrBPh when compared to similarly substituted compounds, such as $4-\mathrm{BrBPh} .{ }^{16} \mathrm{It}$ is demonstrated here that this asymmetry is maintained in both the stable and the metastable phase. The torsion angle with the brominated ring for either stable, $68.3(5)^{\circ}$, and metastable, $66.8(10)^{\circ}$, phases is quite large, when compared to the unsubstituted benzophenone with values around $27^{\circ}$ and $41^{\circ} .{ }^{11}$ Nonetheless, close similarity is found when comparing the central bond angle at the carbonyl group, C1A-C1-C1B, which are $117.8(3)^{\circ}$ and $117.7(7)^{\circ}$ for $2-\mathrm{BrBPh}$ and $121.4(2)^{\circ}$ and $118.9(1)^{\circ}$ for $\mathrm{BPh}$ for the stable and metastable phases, respectively. 
Table 3. Main characteristic torsion angles of the stable and metastable phases of 2bromobenzophenone ${ }^{a}$

\begin{tabular}{lcc}
\hline Torsion Angle & Form I $/{ }^{\circ}(\sigma)$ & Form II $/^{\circ}(\sigma)$ \\
\hline O1-C1-C1A-C2A & $106.4(5)$ & $118.5(8)$ \\
\hline O1-C1-C1A-C6A & $68.3(5)$ & $66.8(10)$ \\
\hline C1B-C1-C1A-C2A & $70.0(5)$ & $65.1(9)$ \\
\hline O1-C1-C1B-C2B & $161.3(4)$ & $152.5(7)$ \\
\hline O1-C1-C1B-C6B & $17.6(6)$ & $28.8(9)$ \\
\hline C1A-C1-C1B-C2B & $22.4(5)$ & $23.9(8)$ \\
\hline C1-C1A-C2A-Br1 & $4.3(5)$ & $4.3(9)$ \\
\hline
\end{tabular}

${ }^{a}$ For the atom labels see the inset of Figure 5.
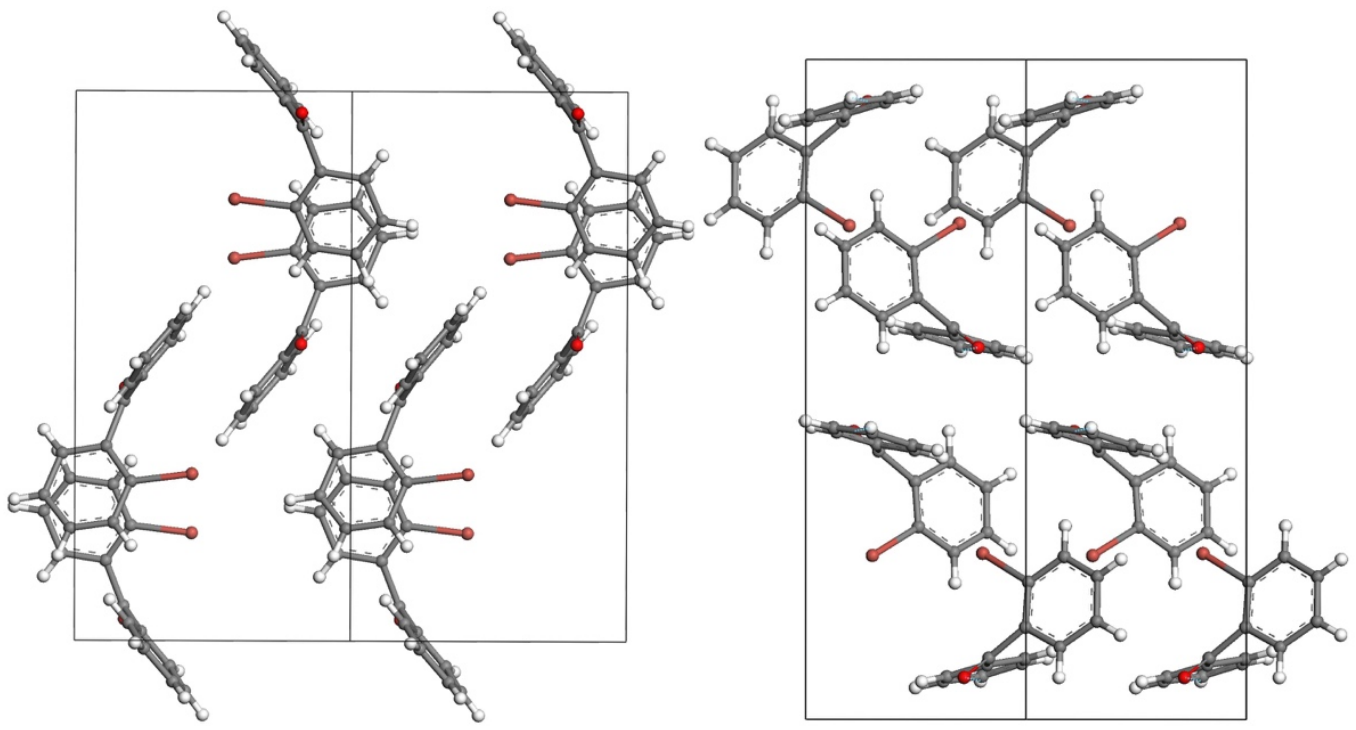

Figure 4. Projections of the 2-bromobenzophenone structure on the $a b$ plane ( $P 2_{1} / a$ setting) or $c b$ plane $\left(P 2_{1} / c\right.$ setting) for the stable phase form I (left) and on the $c b$ plane $\left(P 2_{1} / c\right.$ setting) for the metastable phase form II (right)

As far as the packing is concerned, the structure of the metastable phase is quite different from that of the stable phase. Figure $\mathbf{4}$ contains the structure of both stable and metastable phases projected on the $c b$ plane $\left(P 2_{1} / c\right.$ setting for both structures). Fingerprint plots and Hirshfeld surfaces $^{35}$ are provided in the supplementary materials (Figures $\mathbf{S 4}$ and $\mathbf{S 5}$ ).

Both the stable phase and the metastable phase exhibit aromatic interactions. In the stable phase, form I, a number of strong aromatic interactions can be found (Figures S2a, b, and c). ${ }^{36}$ They are: i) a T-shaped interaction (C4A-H4A $\cdots \pi$ ), also observed by Baumer et al., ${ }^{16}$ with a distance between the hydrogen and the ring of $2.96 \AA$ and a distance of $5.11 \AA$ between the centers of the two rings (Figure S2a), ii) a parallel-displaced interaction between two unsubstituted rings with a center-to-center distance of $4.64 \AA$ and a shortest atom-atom (C4B …C4B) distance of $3.59 \AA$ (Figure S2b), and iii) a second parallel-displaced interaction between two bromine-containing rings with a center-to-center distance of $4.03 \AA$ (Figure S2c). In total three strong and four moderate aromatic interactions (see for example Figure $\mathbf{S 2} \mathbf{d}$ for a moderate interaction) are found in the stable form by the Al aromatic interaction analyzer in 


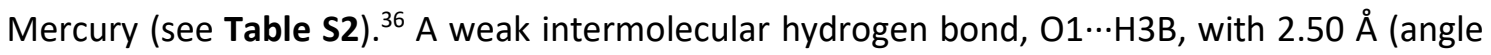
$167.7^{\circ}$ and $\mathrm{O} 1-\mathrm{C} 3 \mathrm{~B}$ distance $3.412 \AA$ ) is present in the structure in combination with an even longer, weaker one, $01 \cdots \mathrm{H} 6 \mathrm{~A}$ (angle $153.0^{\circ}$ and 01 - C6A distance $3.562 \AA$ ), with $2.71 \AA$.

In the structure of the metastable phase, form II, the shortest hydrogen bond distances have shifted to $2.67 \AA$ for $01 \cdots \mathrm{H} 4 \mathrm{~B}$ (angle $166.0^{\circ}$ and 01 - C4B distance $3.580 \AA$ ) ), and $2.680 \AA$ for

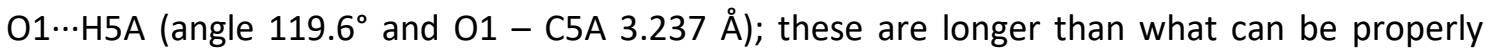
considered as a hydrogen bond. ${ }^{37-40}$ In terms of aromatic interactions, two strong T-shaped interactions exist (Figures S3a and b), one with the C6A-H6A $\cdots \pi$ distance of $2.73 \AA$ Along the $c$ direction and a center-to-center distance of $5.01 \AA$ (Figure S3a) and a second one with a C4A$\mathrm{H} 4 \mathrm{~A} \cdots \pi$ distance of $2.96 \AA$ and a center-to-center distance of $5.14 \AA$ (Figure S3b). In addition, eight moderate T-shaped and parallel-displaced interactions (see for example Figures S3c and d) can be found in the metastable form (see Table S3) by the aromatic interaction analyzer in Mercury. ${ }^{36}$ Thus, although the stable form I possesses more strong interactions, the denser form II (Table 2) compensates the lack of stronger interactions by the presence of eight moderate aromatic interactions (compare Tables $\mathbf{S 2}$ and $\mathbf{S 3}$ ).

Lattice parameters of both forms have been obtained as a function of the temperature (see Supporting Information Table S4) from $100 \mathrm{~K}$ up to the respective melting points. Figure 5 presents the specific volumes of both polymorphs together with that of the liquid phase as a function of the temperature.

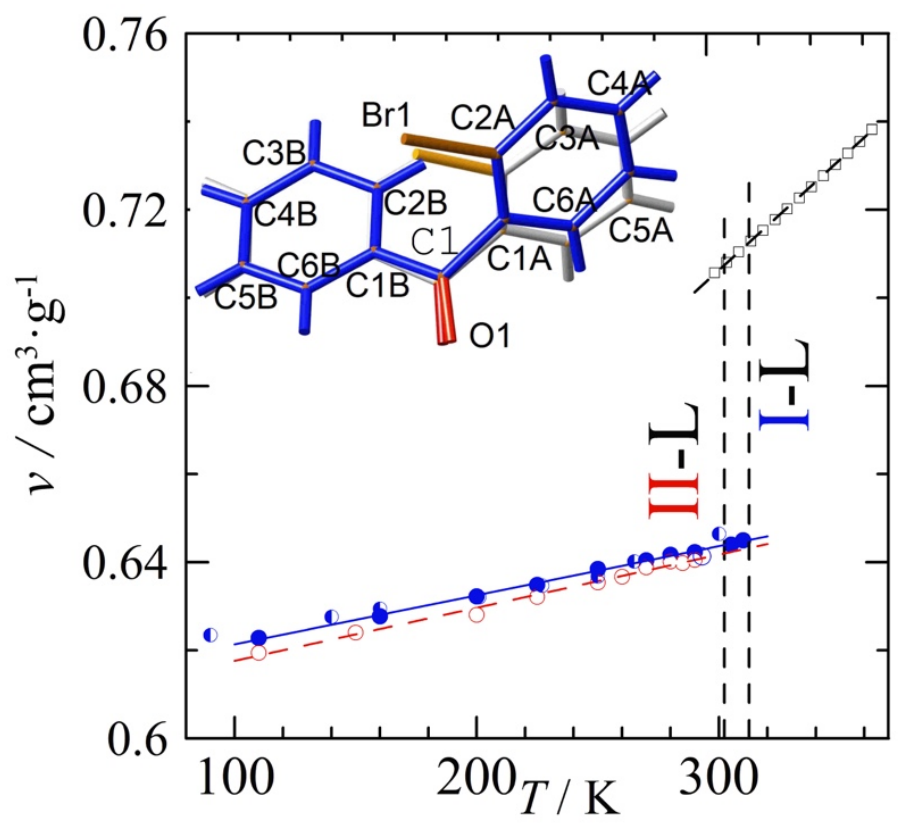

Figure 5. The specific volume of 2-bromobenzophenone as a function of the temperature for the stable form I (solid blue circles, solid line), the metastable form II (open red circles, dashed line), and the liquid phase (open squares, dashed line). The open blue circle corresponds to the single crystal study of Baumer et al. ${ }^{16}$ and half-full circles correspond to the X-ray powder diffraction values of the stable form I from Prokhvatilov et al. ${ }^{41}$ Inset: Molecular conformations with atom labels of 2-bromobenzophenone in the stable form I (grey) and metastable form II (blue). Brown and red bonds correspond to the $\mathrm{C}-\mathrm{Br}$ and $\mathrm{C}=\mathrm{O}$ bonds, respectively. 
The results in Figure $\mathbf{5}$ clearly demonstrate that the specific volume of the stable form I is larger than that of form II at any given temperature, which means that the metastable form II is denser than the stable form I, contrary to the expected behavior according to the density rule of Burger and Ramberger. ${ }^{42}$ This implies that with increasing pressure the relative stability of the denser form II increases and that of form I decreases.

The specific volumes of the three condensed phases have been fitted as a function of the temperature using the least-squares method leading to the following equations:

$v_{1}\left(/ \mathrm{cm}^{3} \mathrm{~g}^{-1}\right)=11.1(6) \cdot 10^{-5} T(/ \mathrm{K})+0.6102(4)$

$v_{\text {II }}\left(/ \mathrm{cm}^{3} \mathrm{~g}^{-1}\right)=12.0(4) \cdot 10^{-5} \mathrm{~T}(/ \mathrm{K})+0.6055(10)$

$v_{\mathrm{L}}\left(/ \mathrm{cm}^{3} \mathrm{~g}^{-1}\right)=50.1(2) \cdot 10^{-5} T(/ \mathrm{K})+0.5559(8)$

With these equations, the volume changes on melting and between the two solids have been determined (Table 1).

\section{Analysis of the thermal expansion}

In order to analyze the anisotropy in the intermolecular interactions, lattice parameters (Table S4) were fitted by a standard least-squares method to a polynomial function of the temperature. The polynomial coefficients describing the temperature dependencies have been compiled in Table S5 in the Supporting Information. The thermal expansion tensor of a monoclinic structure is defined by the principal coefficients, $\alpha_{1}, \alpha_{2}$, and $\alpha_{3}$, which form an orthogonal system, while the axis related to $\alpha_{2}$ is parallel to the unit-cell axis $\boldsymbol{b}^{43}$ The thermal-expansion tensors for the stable form I and the metastable form II are represented in Figure 6 and have also been provided in the form of a table in the supplementary materials with the correlation between the principal tensor directions and the unit-cell axes (Table S6).
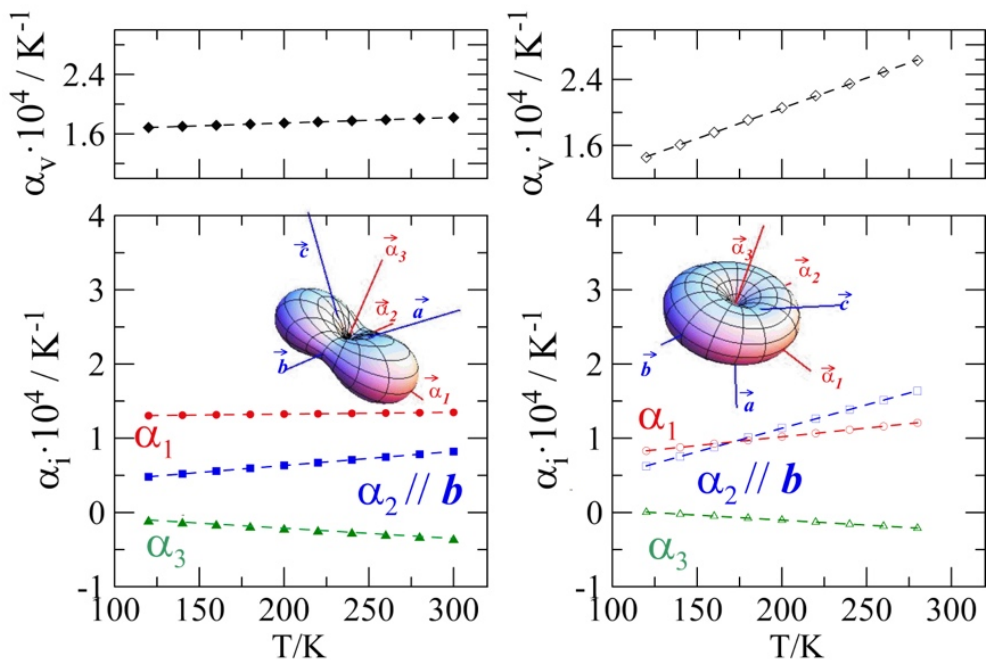

Figure 6. Eigenvalues (bottom) of the thermal-expansion tensor and thermal expansivity $\alpha_{v}$ (top) for stable form I (left) and metastable form II (right) as a function of the temperature. (Insets) The thermal expansion tensor at $200 \mathrm{~K}$ for the stable $\left(P 2_{1} / a\right.$ setting $)$ and metastable $\left(P 2_{1} / C\right.$ setting) phases. 
Figure 6 shows the eigenvalues as a function of the temperature for the two solid phases as well as a pictorial representation of the thermal-expansion tensor at $200 \mathrm{~K}$. Except for the lowest temperatures, the overall expansivity, $\alpha_{v}$, (see upper panels of Figure 6) is higher in the metastable form II than for the stable form I. However, negative eigenvalues, implying contraction on heating and therefore called 'hard direction', ${ }^{43}$ are present in the expansion tensors of both phases ( $\boldsymbol{\alpha}_{3}$, see Figure 6 ).

For the stable phase, the hard direction (within the ac plane) can hardly be a consequence of the $\mathrm{C}-\mathrm{H} \cdots \mathrm{O}$ hydrogen bonds although they run along the [100] direction, because they are simply too weak distance-wise and donor-wise. As can be seen in Table S4, in the supplementary materials, the angle $\beta$ decreases with increasing temperature, while the $a$ and $c$ axes increase in length. This can be interpreted as the unit cell being pulled at its origin (in the $a c$ plane) and at the opposite side of the diagonal $\mathbf{a}+\mathbf{c}$, which within the crystal, translates into two layers moving in opposite directions as indicated in Figure 7a. While this causes expansion along the diagonal $\mathbf{a}+\mathbf{c}$, it diminishes the length of the diagonal $\mathbf{c}-\mathbf{a}$, similar to the situation described by Bhattarcharya and Saha. ${ }^{31}$ However, contrary to the latter paper, in which the crystal contains several strong hydrogen bonds, ${ }^{31}$ the current molecule does not have any hydrogen bonds that could be implicated in NTE and there is no clear reason, why these layers don't expand by moving away from each other. A tentative explanation may be the aromatic interactions, which possess a certain directionality. Although a number of these interactions is located within the moving layers, a strong interaction (according to the aromatic interaction module of Mercury ${ }^{36}$ ) is exactly positioned along the $\mathbf{c}-\mathbf{a}$ direction that experiences the NTE (Figure 7a double red arrow and Figure S2b). It appears that the distance between the centers of the two aromatic cycles indicated by the red double arrow decrease on heating indicating a possible strengthening of this interaction. It is therefore of interest to investigate more precisely the role of the aromatic interactions and their directionality in this particular NTE. 

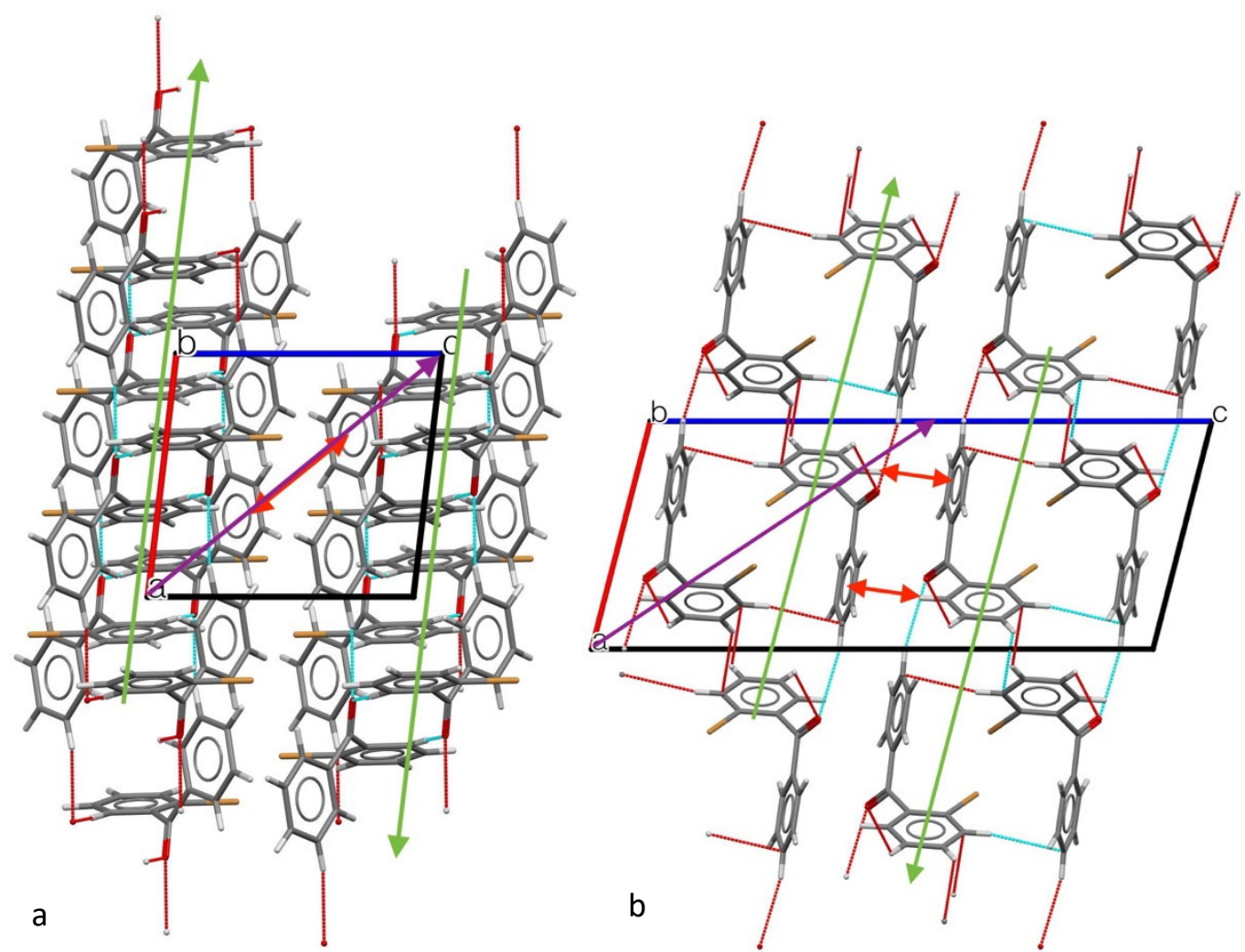

Figure 7. Two layers in (a) form I (ac plane, $P 2_{1} / a$ setting) and (b) form II (ac plane, $P 2_{1} / c$ setting) moving in opposite direction (green arrows) on heating decreasing the angle $\beta$. The NTE is indicated by a purple line, while the strong aromatic interaction is indicated by a red double arrow. The explanation is tentative, in particular for form II, because the NTE displacement is small.

As for the hardest $\boldsymbol{\alpha}_{3}$ direction of the metastable phase, the mechanism is less clear, because there is less movement in the system. However, again in the ac plane layers can be observed that are solely interconnected by aromatic interactions (Figure $\mathbf{7 b}$, T-shaped interactions). As the angle $\beta$ again decreases slightly on heating a similar sliding movement of the layers can be expected. The strongest expansion is along the $b$ axis, which lacks any of the stronger aromatic interactions. As the hydrogen bonds are basically nonexistent in this structure, it would once again be of interest to investigate more closely the role of the aromatic interactions in the observed NTE.

It could be argued that in some way steric hindrance may in part cause the NTE through a trellis effect (as the steric interaction itself would be repulsive and therefore cause expansion). However, due to the small size of the 2-BrBPh molecule, scenarios such as found for the much longer glipizide ${ }^{28}$ are rather unlikely and it is therefore hard to imagine how steric interactions could cause NTE in the present case. In addition, the clear partitioning of both structures in layers makes it less likely that steric hindrance limits these layers from moving away from each other. Possibly, theoretical calculations, such as DFT, preferably with the effect of temperature taken into account, may provide additional information on the expected strength of the aromatic interactions and its role for this molecule in these two structures. 


\section{Relative stability of the crystalline phases}

The high-pressure melting temperatures of both forms are shown in Figure $\mathbf{8}$ for a series of pressures. Several examples of high-pressure thermograms are presented in Figure $\mathbf{S 6}$ for both polymorphs in the Supporting Information. The experimental onset melting temperatures as a function of pressure were fitted with a linear expression leading to:

$T_{\text {I-L }} / \mathrm{K}=0.290(12) P / \mathrm{MPa}+313.4(1.6)$

$T_{\text {II-L }} / \mathrm{K}=0.322(12) P / \mathrm{MPa}+301.2(1.5)$

The experimental slopes, $\mathrm{d} T / \mathrm{d} P$, corresponding to the melting of forms I and II, match perfectly with those calculated according to the Clapeyron equation (see Table 1).

High-pressure thermal analysis results demonstrate that the melting temperatures of the two polymorphs approach each other on increasing pressure as the slope $d T / d P$ of the melting of the metastable form II is steeper than the slope of the higher melting form I, which can be seen in the left-hand panel of Figure 8. In the center panel of Figure 8, the $T$ and $P$ axes have been exchanged in line with most phase diagrams in the pharmaceutical community. The slopes convert therefore into $\mathrm{d} P / \mathrm{d} T$. Due to the inequality $\mathrm{d} P / \mathrm{d} T_{1-\mathrm{L}}>\mathrm{d} P / \mathrm{d} T_{\text {II-L }}$ the melting curves converge at the triple point I-II-L (Figure 8, center panel). This convergence implies that form II possesses a stable domain at high pressure and high temperature above the triple point I-II-L. In addition, because $\Delta S_{I-L}>\Delta S_{I-L}, S_{I}<S_{\|}$and while $v_{1}>v_{\| \mid}$, the slope of the I-II transition $\mathrm{d} P / \mathrm{d} T_{I-I}$ should be negative. Accordingly, the topological pressure-temperature phase diagram corresponds to that depicted in the center panel of Figure 8. In summary, the relationship of form II with respect to form I, which is monotropic at normal pressure and up to the triple point $\mathrm{I}-\mathrm{II} \mathrm{L}$, becomes enantiotropic for temperatures and pressures above this triple point (see the Gibbs-energy diagrams in the right-hand panels in Figure 8).
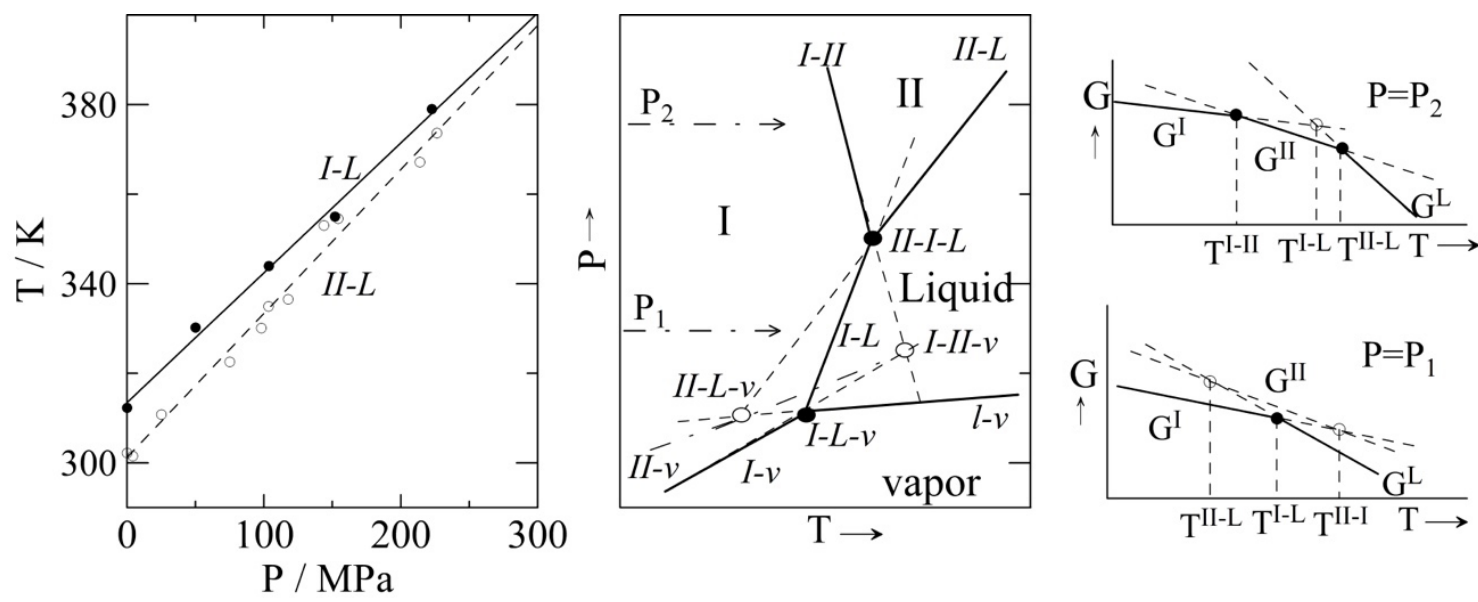

Figure 8. Left-hand panel: Experimental temperature-pressure phase diagram of 2bromobenzophenone with solid and open circles the melting transitions of form I and form II, respectively. Center panel: Topological pressure-temperature phase diagram of 2bromobenzophenone. Right-hand panel: Isobaric sections of the Gibbs-free-energy-vstemperature diagrams at $\mathrm{P}=\mathrm{P}_{1}$ (monotropic behavior, bottom panel), and at $\mathrm{P}=\mathrm{P}_{2}$ (enantiotropic behavior, top panel). Solid lines represent stable equilibria (minimum Gibbs free energy) 
whereas dashed lines represent metastable equilibria. Solid and open circles represent stable and metastable three-phase equilibrium points, respectively.

\section{CONCLUSIONS}

The structure of the metastable form II of 2-bromobenzophenone has been solved in the centrosymmetric monoclinic space group $P 21 / c$ with $a=8.4896(19) \AA, b=6.5438(8) \AA, c=$ $20.253(1) \AA ; \beta=104.452(6)^{\circ}$ and $Z=4\left(Z^{\prime}=1\right)$ and is found to be a racemate.

T-shaped and parallel-displaced aromatic interactions are important in both form I and form II. The latter is the densest of the two even if it is less stable and exhibits a more pronounced thermal expansion. It is striking, however, to observe that both forms exhibit uniaxial contraction on heating. The NTE can in both cases be explained by layers shifting in opposite directions; however, because of the absence of any significant hydrogen bonding, it is of interest to find out to what level the aromatic interactions both in the stable form I and in the metastable form II are responsible for the NTE behavior. In this respect, theoretical calculations, in particular as a function of the temperature, would be a welcome addition to this experimental study.

Thermodynamic properties at normal and high pressure have been determined for the stable and metastable phases and the pressure-temperature phase diagram has been constructed. While the metastable phase behaves monotropically with respect to the stable phase, with an increase in pressure, the phase relationship between the two polymorphs becomes enantiotropic. The solid-solid equilibrium line, which becomes stable at higher pressure, has a negative slope due to the negative difference in volume and it must be rather steep considering the small value of the $d T / d P$ slope listed in Table 1 . This implies that the solid-solid phase transformation is mainly entropy driven.

\section{Acknowledgements}

This work was supported by the Spanish Ministry of Science and Innovation (Grant FIS201782625-P) and the Catalan Government (Grant 2017SGR-42). We thank A. Jezowski of the Institute of Low Temperature and Structure Research, Polish Academy of Sciences for the purification of the 2-bromobenzophenone sample.

\section{References}

1. Kim, J.; Hanna, J. A.; Byun, M.; Santangelo, C. D.; Hayward, R. C., Designing Responsive Buckled Surfaces by Halftone Gel Lithography. Science 2012, 335 (6073), 1201-1205.

2. Schlumpf, M.; Schmid, P.; Durrer, S.; Conscience, M.; Maerkel, K.; Henseler, M.; Gruetter, M.; Herzog, I.; Reolon, S.; Ceccatelli, R.; Faass, O.; Stutz, E.; Jarry, H.; Wuttke, W.; Lichtensteiger, W., Endocrine activity and developmental toxicity of cosmetic UV filters-an update. Toxicology 2004, 205 (1), 113-122.

3. Costa, E. V.; Sousa, E.; Choosang, K.; Singh, S.; Rocha, J.; Lima, R. T.; Pakkong, P.; Ahmed, S.; Vasconcelos, M. H.; Montanari, C. A.; Pinto, M. M., Structure Based Design, Synthesis, and Evaluation of Potential Inhibitors of Steroid Sulfatase. Curr. Top. Med. Chem. 2014, 14 (8), $1033-$ 1044.

4. Cuquerella, M. C.; Lhiaubet-Vallet, V.; Cadet, J.; Miranda, M. A., Benzophenone Photosensitized DNA Damage. Acc. Chem. Res. 2012, 45 (9), 1558-1570. 
5. Wu, S.-B.; Long, C.; Kennelly, E. J., Structural diversity and bioactivities of natural benzophenones. Natural Product Reports 2014, 31 (9), 1158-1174.

6. Suzuki, T.; Kitamura, S.; Khota, R.; Sugihara, K.; Fujimoto, N.; Ohta, S., Estrogenic and antiandrogenic activities of 17 benzophenone derivatives used as UV stabilizers and sunscreens. Toxicol. Appl. Pharmacol. 2005, 203 (1), 9-17.

7. Surana, K.; Chaudhary, B.; Diwaker, M.; Sharma, S., Benzophenone: a ubiquitous scaffold in medicinal chemistry. Medchemcomm 2018, 9 (11), 1803-1817.

8. Hoshino, S.; Suzuki, H., Electroluminescence from triplet excited states of benzophenone. Appl. Phys. Lett. 1996, 69 (2), 224-226.

9. Zhao, B.; Lu, W. Q.; Zhou, Z. H.; Wu, Y., The important role of the bromo group in improving the properties of organic nonlinear optical materials. J. Mater. Chem. 2000, 10 (7), 1513-1517.

10. Gossner, B., VIII. Untersuchung polymorpher Körper. Zeitschrift für Kristallographie Crystalline Materials 1904, 38, 110-122.

11. Kutzke, H.; Klapper, H.; Hammond, R. B.; Roberts, K. J., Metastable $\beta$-phase of benzophenone: independent structure determinations via X-ray powder-diffraction and single crystal studies. Acta Crystallogr. B 2000, 56 (3), 486-496.

12. Vul, E. B.; Lobanova, G. M., Kristallografiya 1967, 12, 411-415.

13. Ma, B.-Q.; Coppens, P., Variable Conformation of Benzophenone in a Series of Resorcinarene-Based Supramolecular Frameworks. Cryst. Growth Des. 2004, 4 (6), 1377-1385.

14. Kutzke, H.; Al-Mansour, M.; Klapper, H., Stable and metastable crystal phases of 4methylbenzophenone. J. Mol. Struct. 1996, 374 (1), 129-135.

15. Gough, K. M.; Wildman, T. A., Hindered internal rotation in benzophenone. J. Am. Chem. Soc. 1990, 112 (25), 9141-9144.

16. Baumer, V. N.; Romashkin, R. V.; Strzhemechny, M. A.; Avdeenko, A. A.; Pyshkin, O. S.; Zubatyuk, R. I.; Buravtseva, L. M., 2-Bromobenzophenone. Acta Crystallogr. 2005, E61 (4), o1170-o1172.

17. Baran, J.; Davydova, N. A.; Drozd, M., Discovery of a new polymorphic phase of orthobromobenzophenone. Chem. Phys. Lett. 2015, 621, 18-21.

18. Baraldi, I.; Gallinella, E.; Momicchioli, F., Conformations and internal rotation properties of molecules containing one geminal diphenylgroup: diphenylethylene, diphenylketimine, benzophenone, diphenylether and diphenylmethane. J. Chim. Phys. 1986, 83, 653-664.

19. Pinkus, A. G.; Klausmeyer, K. K.; Feazell, R. P.; Meng, L. Y. C.; Chang, T. C., Crystal structures of 2-chloro- and 2,5-dichloro-benzophenones. J. Chem. Crystallogr. 2004, 34 (9), 637640.

20. Strzhemechny, M. A.; Baumer, V. N.; Avdeenko, A. A.; Pyshkin, O. S.; Romashkin, R. V.; Buravtseva, L. M., Polymorphism of 4-bromobenzophenone. Acta Crystallogr., Sect. B: Struct. Sci. 2007, 63 (2), 296-302.

21. Wallach, O., Zur Kenntniss der Terpene und der ätherischen Oele. Justus Liebigs Ann Chem 1895, 286 (1), 90-118.

22. Hunter, C. N.; Sanders, J. K. M., The nature of $\pi-\pi$ interactions. J. Am. Chem. Soc. 1990, 112 (14), 5525-5534.

23. Salonen, L. M.; Ellermann, M.; Diederich, F., Aromatic rings in chemical and biological recognition: energetics and structures. Angew Chem Int Ed Engl 2011, 50 (21), 4808-42.

24. Martinez, C. R.; Iverson, B. L., Rethinking the term "pi-stacking". Chemical Science 2012, $3(7)$.

25. Grimme, S., Do special noncovalent pi-pi stacking interactions really exist? Angew Chem Int Ed Eng/ 2008, 47 (18), 3430-4.

26. Gajda, R.; Domański, M. A.; Malinska, M.; Makal, A., Crystal morphology fixed by interplay of $\pi$-stacking and hydrogen bonds - the case of 1-hydroxypyrene. CrystEngComm 2019, 21 (11), 1701-1717. 
27. Saraswatula, V. G.; Sharada, D.; Saha, B. K., Stronger $\pi \cdots \pi$ Interaction Leads to a Smaller Thermal Expansion in Some Charge Transfer Complexes. Cryst. Growth Des. 2017, 18 (1), 52-56. 28. Burley, J. C., Structure and intermolecular interactions of glipizide from laboratory X-ray powder diffraction. Acta Crystallogr B 2005, 61 (Pt 6), 710-6.

29. Hutchins, K. M.; Groeneman, R. H.; Reinheimer, E. W.; Swenson, D. C.; MacGillivray, L. R., Achieving dynamic behaviour and thermal expansion in the organic solid state via cocrystallization. Chem Sci 2015, 6 (8), 4717-4722.

30. Bhattacharya, S.; Saha, B. K., Steric guided anomalous thermal expansion in a dimorphic organic system. CrystEngComm 2014, 16 (12).

31. Bhattacharya, S.; Saha, B. K., Uniaxial Negative Thermal Expansion in an Organic Complex Caused by Sliding of Layers. Cryst. Growth Des. 2012, 12 (10), 4716-4719.

32. Würflinger, A., Differential thermal-analysis under high-pressure IV. Low-temperature DTA of solid-solid and solid-liquid transitions of several hydrocarbons up to $3 \mathrm{kbar}$. Ber. BunsenGes. Phys. Chem. 1975, 79 (12), 1195-1201.

33. Neumann, M. A., X-Cell: a novel indexing algorithm for routine tasks and difficult cases. J. Appl. Crystallogr. 2003, 36, 356-365.

34. Pawley, G., Unit-cell refinement from powder diffraction scans. J. Appl. Crystallogr. 1981, 14 (6), 357-361.

35. Turner, M. J.; McKinnon, J. J.; Wolff, S. K.; Grimwood, D. J.; Spackman, P. R.; Jayatilaka, D.; Spackman, M. A. CrystalExplorer17 https://hirshfeldsurface.net. , v17; University of Western Australia: 2017.

36. Macrae, C. F.; Sovago, I.; Cottrell, S. J.; Galek, P. T. A.; McCabe, P.; Pidcock, E.; Platings, M.; Shields, G. P.; Stevens, J. S.; Towler, M.; Wood, P. A., Mercury 4.0: from visualization to analysis, design and prediction. J. Appl. Crystallogr. 2020, 53 (1), 226-235.

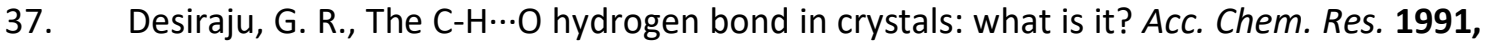
24 (10), 290-296.

38. Steiner, T.; R. Desiraju, G., Distinction between the weak hydrogen bond and the van der Waals interaction. Chem. Commun. 1998, (8), 891-892.

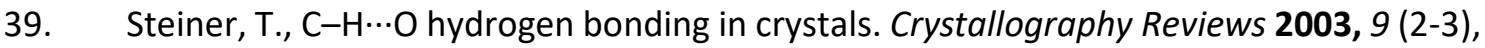
177-228.

40. Desiraju, G. R.; Steiner, T., The Weak Hydrogen Bond In Structural Chemistry and Biology. Oxford University Press: Oxford (UK), 1999.

41. Prokhvatilov, A. I.; Strzhemchny, M. A.; Galtsov, N. N.; Pyshkin, O. S.; Buravtseva, L. M.; Aksenova, N. A., Lattice parameters and thermal expansion of 2-bromobenzophenone crystals in the 90-300 K range. Low Temperature Physics 2016, 42 (4), 317-320.

42. Burger, A.; Ramberger, R., Polymorphism of Pharmaceuticals and Other MolecularCrystals .1. Theory of Thermodynamic Rules. Mikrochim. Acta 1979, 2 (3-4), 259-271.

43. Salud, J.; Barrio, M.; Lopez, D. O.; Tamarit, J. L.; Alcobe, X., Anisotropy of intermolecular interactions from the study of the thermal-expansion tensor. J. Appl. Crystallogr. 1998, 31, 748757. 
For Table of Contents only:

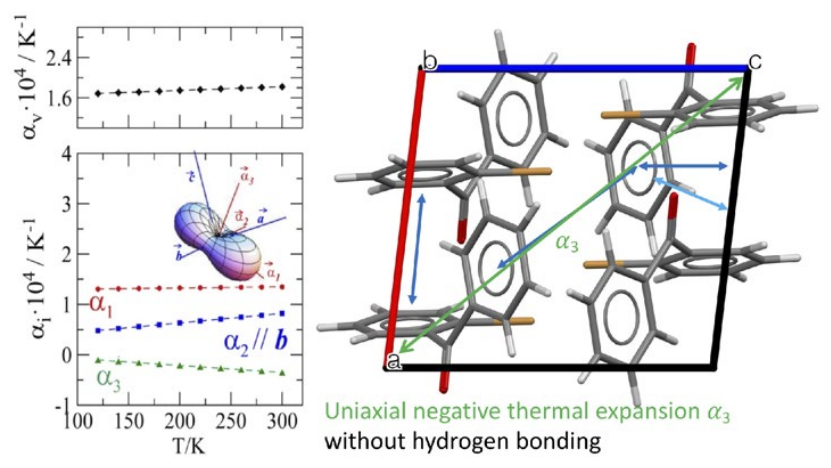

